\title{
Ventilator Dependency in ALS: Management, Disease Progression, and Issues of Coping
}

\author{
${ }^{1}$ Mark B. Bromberg, MD, PhD, 1 Dallas A. Forshew, RN, BSN, \\ 2 Sandra Iaderosa, MSW, ${ }^{3}$ Evelyn R. McDonald, RN, MS
}

\begin{abstract}
The natural progression of amyotrophic lateral sclerosis (ALS) leads to respiratory failure and death. Artificial ventilation can prolong the course, leading to extreme degrees of weakness and dependence. Little specific information is available to counsel ALS patients about making the decision for artificial ventilation. In order to gain more information, we visited four ventilator-dependent ALS patients and their primary caregivers. We determined the neurologic state and level of function of the patients and interviewed their primary caregivers to assess medical care and management needs (both social and financial) and how they were being met. We also administrated questionnaires to assess the psychological well-being of both patient and primary caregiver and how the relationship between the patient and primary caregiver changed under these circumstances. Key Words: ALS-Artificial ventilation-Disease progression.
\end{abstract}

\section{Introduction}

Amyotrophic lateral sclerosis (ALS) is the most common form of motor neuron disease in adults. It is characterized by progressive muscular weakness, and the natural coutse leads to respiratory failure and death. Ventilator support can artificially extend the course of the disease and the life of the patient for an indefinite period. At some point in the natural course of ALS, patients and primary caregivers need information to aid them in making a choice about ventilator support that will lead either to a comfortable natural death or to a satisfactory quality of life on a ventilator.

From the I Department of Neurology, University of Utah, Salt Lake Ciry, Utah; ${ }^{2}$ Department of Social Work, University of Michigan, Ann Arbor, Michigan; ${ }^{3}$ Department of Research and Education, The New Road Map Foundation, Seattle, Washington.

Address correspondence to: Dr. Mark Bromberg, Department of Neurology, University of Utah, 50 North Medical Drive, Salt Lake City, UT 84132, phone (801) 581-6873, fax (801) 581-4192.
The management of issues surrounding respiratory failure is a challenge for the patient, the primary caregiver, and health care providers (1). The current study evolved from our experiences in a motor neuron disease clinic with ALS patients faced with life or death decisions. Although there are reports that address factors important in the decision process and identify practical aspects of artificial ventilation (2-4), many questions arise for which there is limited information to guide health care providers. Drawing from our clinic experience, we identified a number of specific questions we considered important for our patients to help them make decisions. To provide full answers, we needed more information and designed this study to interview ventilator-dependent ALS patients and their primary caregivers. Although we had specific questions to address, we remained open to issues that were important to the patients and caregivers.

Our goals in this pilot study were to acquire information about the progression of ALS and issues of management during the extended natural history, to identify issues of importance to the patient and primary caregiver, and to develop effective instruments for a larger study. An additional goal was to highlight effective ways for the 
health care provider to assist patients and primary caregivers in making the very personal decision about ventilator support.

\section{Methods}

We studied four ALS patients contacted through the Forbes Norris MDA/ALS Center, San Francisco, California, who had ALS and were dependent on artificial ventilation and lived at home. The patients and their primary caregivers were interviewed in their homes by the authors. The interview consisted of a series of questions, questionnaires, and a neurologic examination. Primary caregivers were usually the spouse, but if there was no spouse or significant individual, some questions were omitted. The following topics were addressed. The questionnaires are reproduced in the appendix.

(1) The course of ALS before respiratory failure and the subsequent pattern and rate of progression of weakness were determined. Emphasis was placed on communication abilities. A neurologic examination was performed, and contributing factors from other medical conditions were sought.

(2) An inventory of medical care issues and their management was made, with emphasis on daily care, nutrition, and access to medical care.

(3) The extent of daily activities was identified, and the ALS Functional Rating Scale was administered. The range of the scale was 0 (total dependence for all daily functions) to 50 (total self-sufficiency with no limitations). The frequency of visits outside the home and the distance traveled from the home were assessed. We also determined limitations on activities of the primary caregivers due to the need to provide assistance for the patient.

(4) Questions about quality of life were addressed to the patient and caregiver, including what changes were attributed to artificial ventilation, whether the patient would choose artificial ventilation again, whether the caregivers would choose it for themselves, and how the caregivers thought that the patients' lives would end.

(5) The patient's psychological well-being was asșessed using three standardized tests previously found to be predictive of the psychological status of ALS patients (5): the Beck Depression Inventory (6) (depression level: 0-9 none, 10-18 mild to moderate, 19-29 moderate to severe, 30-63 severe); the Health Locus of Control (7) (11 most internal, 66 most external); and the Purpose in Life test $(8)$ ( $<92$ lack of clear purpose, $92-112$ indecisive, $>112$ definite purpose). A composite "Worldview Score" was derived from the individual scores (Purpose in Life score minus Health Locus of Control score minus Beck Depression Inventory score). Conceptually, Worldview measures a person's view of self and their world, their purpose in life, and whether they experience their health as internally or externally controlled. The Worldview Score range is -109 to +129 . Worldview scores represent "positive" $(\geq+75)$, "neutral" $(+37$ to +74$)$, and "negative" $(\leq+36)$ Worldview (9). Higher scores of this composite variable reflect a positive Worldview, while lower scores reflect a negative Worldview.

(6) The circumstances surrounding ventilator support and how the choice was made were explored, including whether adequate information was available both for making the choice and for ventilator management. Financial issues and availability of community resources were inquited about.

(7) The relationship between patient and primary caregiver and any changes in that relationship related to artificial ventilation were explored. Stress and mechods of coping with the emotional aspects of ALS were identified.

\section{Results}

This was a pilot study, and statistically meaningful conclusions were not anticipared. Only selected findings that raise noteworthy issues are presented.

\section{Demographic Features}

Three patients were male and one female. The average age of ALS symptom onset was 59 years (range 31 to 67 years) and the average time using artificial ventilation was 6 years (range 2 to 12 years). Ventilator support was instituted early in two patients who experienced respiratory failure as their presenting symptom, and was required unexpectedly in a third because of acute respiratory failure. One patient had a possible second medical condition influencing his current neurologic state: a cognitive decline thought to be related in part to presumed anoxia during the initial respitatory failure, or to an ALS-related dementia, or to both factors.

\section{Neurologic Examination}

The neurologic examination revealed progressive weakness in all patients. In two patients there was complete paralysis of muscles in all limbs, with only palpable contractions in one or two muscles in each patient. 
Two patients could stand to assist in transfers, but required support for stability. One of these had ALS for two years and retained good upper limb function and could write well. The other patient had rapid paralysis of upper limb muscles during the first two years but could still support weight after being on a ventilator for 12 years of ALS. In contrast to the marked degree of limb paresis or total paralysis, all patients had some movements of muscles of facial expression, which aided communication. Limitations of extraocular motility and abnormal eye movements (saccadic pursuits and impersistence) were observed only in the patient with cognitive difficulties. The other three patients had reduced vibratory sensation perception (128 $\mathrm{Hz}$ tuning fork).

\section{Communication}

The ability to communicate varied among parients. None could communicate by voice, and lip reading by an unfamiliar visitor was not possible. One patient could communicate slowly but easily by writing, and another could communicate with effort and patience by Morse code or by computer. These two patients were writing their memoirs. Meaningful communication with the patient with cognitive difficulcies was not possible beyond simple mimicry needed for the neurologic examination.

\section{Medical Care}

The initial diagnosis and medical care were obtained in specialty clinic settings, but reduced mobility due to disease progression limited patient access to medical care. Routine visits to health care providers were less frequent than social visits, ranging from rare (once per year) to none over several years. Patients took from three to eleven medications for ALS-related conditions, including medications for excess saliva, sleep, constipation, labile emotions, and anxiety. Caregivers described few medical problems requiring treatment. Several primary caregivers were able to diagnose medical conditions, and after telephone consultation with a physician treatment was approved and prescribed in this remote manner.

\section{Home Care}

All patients were cared for at home with professional help assisting between 18 and 24 hours per day. One patient had no spouse or consistent primary caregiver. Two of the primary caregivers assisted with care by taking one shift. Only one primary caregiver had a full-time profession. The ALS function scores were very low, with an average value of 3 (range 0 to 7). Accordingly, all patients were confined to chair or bed. Musculoskeletal pain from an inability to voluntarily shift position, especially at night, was not described. All parients required permanent feeding tubes for all nutrition and fluids, and their weight remained stable. For some, bowel regularity was problematic. There were no major problems with ventilator care, either mechanically or from the patient care perspective. All patients were able to leave the house for short trips and longer interstate vacations. Travel became harder as weakness progressed, and trips were reduced in frequency. Home visits by friends and family were frequent for all patients.

\section{Stresses, Coping Mechanisms, and Quality of Life}

Full insurance coverage was rare, and the financial burden of home ventilator care was a significant stress for most families. This required the disposition of personal items, including the sale of a house in one instance. These financial sacrifices were not a factor in the decision to remain on a ventilator. Inability to communicate and lack of mobility were the most stressful issues for the patient. For primary caregivers, major issues were the inability to influence disease progression and guilt at being able-bodied. When spouses were the primary caregivers, there was fear of being left alone to handle a ventilator emergency. Friends were not comfortable tending patients alone and brief respites for the spouse were not possible without professional help. Support groups were helpful, but lack of patient mobility prevented attendance by patients and primary caregivers. Primary caregivers felt that support groups were more helpful before respiratory failure and that issues particular to ventilator patients were "beyond" the usual support group format and content. Religion-and spiritulality were sources of support for most patients. Social agencies, including insurance companies, were felt to be responsive.

\section{Psychological Well-Being}

For the three people who could be tested, Purpose in Life scores ranged from 98 to 118, Health Locus of Control from 30 to 39, and Beck Depression Inventory from 9 to 14 . Worldview score ranged from 49 to 79 . Two patients had a neutral Worldview and one patient had a positive Worldview. One patient felt that quality of life markedly deteriorated when placed on a ventilator, one felt it improved, and one saw little change. 


\section{Discussion}

ALS is a unique disease because the rate of progression for most patients is slow, providing an opportunity to make decisions regarding artificial ventilation (1). Patients and caregivers may find it hard to hear and absorb information that will help them make an informed decision, even when it is presented in a timely and thorough manner. This suggests that the topic needs to be revisited frequently.

Although rare, respiratory failure can be the presenting or early symptom of ALS $(10,11)$. Two patients we interviewed presented in this way, and a third patient had acute respiratory failure later in the disease process. It is important to emphasize that respiratory failure in ALS, whether as the first symptom or later in the course of the disease, frequently presents as an urgent medical problem. In a recent survey, $58 \%$ of ventilator-dependent ALS patients were intubated under emergent conditions (2). The issue of dissemination of information under these conditions was mentioned as a problem by several caregivers in our study. It may be difficult to provide full information when respiratory failure is the presenting symptom of ALS and the diagnosis is not reached until after artificial ventilation has been started.

When there is pressure to make a decision, we suggest that consideration be given to BiPAP or CPAP, as an alternative form of temporary and noninvasive assisted ventilation. With a full understanding that these devices are only assistive and are not a substitute for positive pressure tracheal ventilation, they allow a patient and caregiver to obtain some practical experience with assisted ventilation and give them time to absorb adequate information to make an informed decision. With respiratory failure as the presenting symptom, especially if ALS is suspected, temporary assisted ventilation gives time to complete the diagnostic evaluation and to provide thorough information.

One patient in this study who had been on artificial ventilation for twelve years had an initial two-year period of rapid progression of loss of arm strength; followed by a much slower loss of leg strength over the next ten years. Despite total upper extremity paralysis during this twelveyear period, he had sufficient cervical motor neurons to permit independent breathing for up to four hours a day. Although this patient had a markedly nonlinear rate of progression of different muscle groups, while he was losing arm strength, severe bulbar weakness impeded his nutritional intake, and his body weight dropped approximately $40 \%$ before it stabilized and increased with placement of a feeding tube. This raises the question of whether malnutrition can play a role in rapid rates of loss of strength and emphasizes the role of adequate nutrition in ALS (12).

Although ALS symptoms revolve around loss of motor neurons, subclinical involvement of other neuronal systems can be shown, especially in patients who have had prolonged ventilator support (13). In the somatosensory system, neuronal degeneration can be found at postmortem examination (13-15), and a loss of sensory fibers can be demonstrated by serial electrodiagnostic studies (16). Quantitative sensory testing in non-ventilator-dependent patients shows a statistically significant elevation in the threshold for detection of a vibrating stimulus (17), and we found a readily detectable vibratory sensory loss with a hand-held tuning fork in ventilator-dependent patients. None of the patients interviewed listed musculoskeletal pain as a major problem, which contrasts to our clinical experience and that of others with patients during the natural course of ALS (18) when pain from immobility is a frequent complaint. The lack of pain may be associated with the loss of neurons in the somatosensory system. It may be reassuring that as immobility becomes complete and the ability to communicate falls, ventilator-dependent patients may experience less physical discomfort (Bromberg, 1994).

We found that the care of ALS patients on a ventilator can be readily accomplished at home. The burden of management falls on the primary caregiver, most often the spouse (2), but other people can effectively substitute. The spouses we incerviewed were concerned with poor access to information about patient management. Initially, support groups were a source of information before ventilation was started. The small number of ALS pacients on a ventilator, $5 \%$ of ALS patients in a sample region of the United States (2), means that support groups have less to offer, and spouses believed that they had "outgrown" the group. We propose periodic special support group meetings for ALS ventilator patients, their spouses and caregivers, perhaps held at regional ALS/Motor Neuron Disease clinics or via closed circuit television. Tapes of the proceedings could be made available.

Another issue mentioned by the spouses was freedom to leave the house. A recent survey of ventilatordependent ALS patients found that it takes an average of 3.5 people per day to care for a patient (2). With suitable help, spouses can maintain a professional career.

In spite of the mechanical complexity of artificial ventilation and the physical difficulties moving a paralyzed individual, the patients we interviewed were able to travel long distances for vacations. The effort involved limited the frequency of travel, particularly for short distances, such as for clinic visits. 
Health care was reduced or curtailed because of the effort needed to travel to a clinic, and the burden of medical management shifted to the primary caregiver. In our small sample, the patients were healthy, and problems such as infections could be readily identified by the caregivers, verified at a distance by a local physician, and treated effectively by the caregiver. Neurologic evaluation was virtually absent.

Our patients and primary caregivers were, in general, satisfied with home ventilation, in agreement with the findings by Moss and coworkers (2). Individual psychological test scores indicated that only one patient lacked purpose and meaning, and none were severely depressed. Contrary to our expectations, none of the patients exhibited a negative Worldview score. Major concerns expressed by patients were immobility and lack of communication abilities. As with ALS patients not on ventilator support (19), the level of function did not predict a patient's satisfaction with life.

In summary, we have completed a pilot study of ventilator-dependent ALS patients and their primaty caregivers. A unique feature of this study is a team that included a neurologist, a nurse, and a social worker, each gathering specific types of information. Findings confirmed a continued progression of weakness, but the rate may not be linear in every patient, and loss of sensory neurons may help a patient be more comfortable despite being immobile. These patients had limited access to medical care. Poor dissemination of information about respiratory failure is a major clinical problem, and a dearth of support groups for patients on artificial ventilation is vexing for primary caregivers. Despite these issues, some of our patients are satisfied with their quality of life, and none displayed a negative Worldview score:

Acknowledgment: We thank Dr. Deborah Gelinas, Sheila Brennan, RN, and Dee Holden Norris, RN, of the Forbes Norris MDA/ALS Center, California Pacific Medical Center, San Francisco, California, for providing patients for our study. We thank Drs. Robert Miller and Deborah Gelinas for their review of the manuscript.

Presented in part at the Fifth International Symposium on ALS/MND, 7-9 November 1994, Noordwijk, Holland.

\section{References}

1. Bromberg MB. Life support: realities and dilemmas. In: Mitsumoto $\mathrm{H}$, Norris FH (eds.). Amyotrophic lateral sclerosis: a comprehensive guide to management. New York: Demos, 1994:167-82.

2. Moss AH, Casey P, Stocking CB, Roos RP, Brooks BR, Siegler M. Home ventilation for amyotrophic lateral sclerosis patients: outcomes, costs, and patient, family, and physician acticudes. Neurology $1993 ; 43: 438-43$.

3. Sivak ED, Gipson WT, Hanson MR. Long-term management of respiratory failure in amyorrophic lateral sclerosis. Ann Neurol $1982 ; 12: 18-23$.

4. Silverstein MD, Stocking CB, Antel JP, Beckwith J, Roos RP, Siegler M. Amyotrophic lateral sclerosis and life-sustaining therapy: patients' desires for informanion, participation in decision making, and life-sustaining therapy. Mayo Clin Proc 1991;66:906-13.

5. McDonald ER. Psychosocial-spiritual overview. In: Mitsumoto $H$, Norris FH (eds.). Amyotrophic lateral sclerosis: a comprehensive guide to management. New York: Demes, 1994:205-27.

6. Beck AT, Rush AJ, Shaw BE, et al. Cognitive therapy of depression. New York, Guilford Press, 1979:425.

7. Wallston BS, Wallston KA, Kaplan GD, Maides SA. Development and validation of the Health Locus of Control (HLC) scale. J Consult Clin Psychol 1976;44:580-85.

8. Reker GT, Cousins JB. Factor structure, construct validity and reliability of the Seeking of Noetic Goals (SONG) and Purpose in Life (PIL) tests. J Clin Psychol 1979;35:85-91.

9. McDonald ER. Quality of life from the patient's perspective. J Nexcesci Nurs 1995;27:222-23.

10. Fromm GB, Wisdon PJ, Block A]. Amyorrophic lareral sclerosis presenting with respiratory failure. Chest 1977;71:612-14.

11. Hill R, Martin J, Hakin A. Acute respiratory failure in motor neuron disease. Arch Neutrol 1983:40:30-32.

12. Mazzini L, Corra T, Zaccala M, Mora G, Del Piano M, Galance M. Percutaneous endoscopic gastrostomy and enteral nucricion in amyotrophic lateral sclerosis. I Neurol 1995;242:695-98.

13. Hayashi $\mathrm{H}$, Kato $\mathrm{S}$. Total manifestations of amyotrophic lateral sclerosis. J Neurol Sci 1989;93:19-35.

14. Dyck PJ, Stevens JC, Mulder DW, Espinosa RE. Frequency of nerve fiber degeneration of peripheral motor and sensory neurons in amyotrophic lateral sclerosis. Neurology 1975;25:781-85.

15. Bradley WG, Good P, Rasool CG, Adelman LS. Morphometric and biochemical studies of peripheral nerves in amyotrophic lateral selerosis. Ann Neurol 1983; 14:267-77.

16. Mondelli M, Rossi A, Passero S, Gauzzi GC. Involvement of peripheral sensory fibers in anyotrophic lateral sclerosis: electrophysiological study of 64 cases. Muscle Nerve 1993;16:166-72.

17. Mulder DW, Bushek W, Spring E, Karnes J, Dyck PJ. Motor neuron disease (ALS): evvaluation of detection thresholds of cutaneous senșation. Neurology 1983;33:1625-27.

18. Newrick PG, Langton-Hewer R. Pain in motor neuron disease. J Neurol Nearosurg Psychiatry 1985;48:838-40.

19. McDonald ER, Wiedenfeld $S A$, Hillel A, Carpenter $\mathrm{CL}$, Walter RA. Survival in amyotrophic lateral sclerosis: the role of psychological factors. Arch Neurol 1994;51:17-23. 